\title{
Elever des porcs dans une ville d'Afrique de l'Ouest : arrangements entre acteurs pour gérer la proximité ville-élevage
}

\author{
Ophélie Robineau ${ }^{1,2}$
}

Mots-clés

Porcin, petite exploitation agricole, agriculture urbaine, pratiques agricoles, alimentation des animaux, Burkina Faso

Submitted: 18 July 2017

Accepted: 15 November 2017

Published: 9 July 2018

DOI : $10.19182 /$ remvt.31288

\begin{abstract}
Résumé
Alors qu'un nombre croissant d'études s'intéressent à l'agriculture urbaine, l'élevage urbain de porcs reste méconnu. Pourtant, il est présent dans de nombreuses villes du Sud et procure un revenu à de nombreuses familles. Cette étude visait à dépasser les analyses centrées sur des aspects techniques de ce type d'élevage pour s'intéresser plus largement au cadre social et spatial dans lequel des éleveurs élèvent des porcs en ville. Elle reposait sur une démarche empirique qui a permis de saisir les conditions du développement et du maintien de l'élevage de porcs dans la deuxième ville du Burkina Faso, Bobo-Dioulasso. Dans cet article, il est question des pratiques des éleveurs à petit effectif, et plus particulièrement du rôle que jouent les arrangements entre acteurs dans la mobilisation des ressources à la fois techniques et sociales qui sont nécessaires à la conduite et au maintien de ce type d'élevage. Les résultats soulignent que les éleveurs de porcs ont une logique de mobilité réduite : les arrangements qu'ils développent avec d'autres acteurs se basent sur une mise en invisibilité de l'élevage à la fois politique, spatiale et sociale, et les liens qu'ils développent pour accéder à des ressources externes sont ancrés dans le temps et dans un voisinage proche. L'environnement social et spatial au sein duquel ils évoluent est central pour la conduite quotidienne des élevages autant que pour leur pérennité à court et moyen terme.
\end{abstract}

- Comment citer cet article: Robineau O., 2018. Rearing pigs in a West African city: Arrangements between actors to manage the proximity between the city and livestock farming. Rev. Elev. Med. Vet. Pays Trop., 71 (1-2): 23-31, doi: 10.19182/remvt.31288

\section{INTRODUCTION}

La question de la « légalité » ou inversement de la tolérance de l'agriculture urbaine se pose dans beaucoup de pays, et la pratique de l'élevage en ville est particulièrement remise en cause du fait des nuisances sonores, olfactives, et des risques de zoonoses induits par la proximité entre hommes et animaux (Boussini et al., 2012). Le maintien ou le développement des maladies traditionnelles comme les helminthes et autres vers, puis les épisodes de grippe aviaire et ensuite de grippe porcine - peurs sanitaires d'ampleur mondiale - ont confirmé les réticences des autorités urbaines vis-à-vis de la présence d'animaux en ville. Pourtant, l'élevage y est toujours présent : l'International Livestock Research Institute (ILRI) a souligné, sans pour

1. CIRAD, UMR INNOVATION, F-34398 Montpellier, France.

Email : robineauophelie@gmail.com

2. INNOVATION, Univ Montpellier, CIRAD, INRA, Montpellier SupAgro, Montpellier, France. autant pouvoir la chiffrer précisément, l'importance de la présence de lélevage urbain dans les villes africaines et son rôle économique pour les ménages à faibles revenus (ILRI, 2012).

Le porc revêt une image particulièrement négative en raison de son odeur, de ses cris et des significations religieuses qui lui sont attachées. Pourtant, son élevage est répandu dans les villes du Sud (Mopaté et al., 2006 ; Chaléard et al., 2014). Au Burkina Faso, l'élevage urbain de porcs en claustration s'est fortement développé durant les dernières décennies (Kiendrebeogo et al., 2012 ; Robineau, 2013). A Bobo-Dioulasso (700 000 habitants), malgré l'interdiction officielle d'élever des porcs en ville depuis la publication d'un arrêté en 1964, la direction provinciale du ministère des Ressources animales a estimé qu'il existe plus de 500 porcheries traditionnelles dans la ville, en opposition aux porcheries plus modernes qui existent dans la frange urbaine. Le « porc au four »- viande grillée consommée dans des rôtisseries de rue -, fait partie des identités culinaires de la ville. Cependant, malgré son importance économique et alimentaire, l'élevage urbain de porcs en claustration est invisible aux yeux de celui qui ne s'y intéresse pas et reste peu documenté, contrairement 
à d'autres formes d'agriculture et d'élevage urbains ( $c f$. par exemple, l'article d'Amadou et al., 2012, qui traite de l'élevage urbain à BoboDioulasso où l'élevage de porcs n'est pas mentionné).

Les données sur l'élevage de porcs en claustration étant peu nombreuses et plutôt axées sur des aspects techniques (Kiendrebeogo et al., 2012 ; Gomgnimbou et al., 2014), l'auteure a entrepris de comprendre ce que veut dire «élever des porcs en ville », et de questionner la façon dont les éleveurs de porcs urbains parviennent à conduire des élevages au cœur même des villes, malgré les réglementations sanitaires, les contraintes d'accès à l'alimentation et la perception négative de ces bêtes. Dans cet article, il est question des pratiques des éleveurs et plus particulièrement du rôle que jouent les arrangements entre acteurs dans la mobilisation des ressources à la fois techniques et sociales - notamment l'alimentation, les mâles reproducteurs, l'information technique et la tolérance de la part des acteurs urbains - qui sont nécessaires à la conduite et au maintien de ce type d'élevage.

\section{MATERIEL ET METHODES}

\section{Approche conceptuelle : durabilité de l'agriculture et arrangements entre acteurs}

La durabilité de l'agriculture a été abordée par certains auteurs sous l'angle des stratégies qu'adoptent les agriculteurs face aux évolutions du contexte économique, politique, environnemental et social. Ceci renvoie à des critères technico-économiques et aux modalités de mobilisation des ressources ; c'est ce que Godard et Hubert (2002) ont nommé la durabilité « autocentrée », qu'ils proposent comme l'une des deux composantes de la durabilité de l'agriculture. L'autre composante, plutôt « exogène », est liée aux effets du territoire sur la durabilité de l'agriculture et vice versa. Ces deux composantes de la durabilité ont été reprises par des agronomes pour questionner les modalités de transformation et d'adaptation des activités agricoles en milieu urbain (Aubry et al., 2008) : les auteurs insistent sur le rôle que joue la durabilité exogène dans le maintien de l'agriculture urbaine. En effet, l'agriculture urbaine n'est pas durable en soi, elle est vulnérable en raison du contexte dans lequel elle se développe (Cantor, 2010). La durabilité exogène est définie entre autres comme les effets qu'ont les dynamiques urbaines sur cette agriculture. Cette double entrée apparaît fertile pour comprendre les conditions du développement et du maintien de l'élevage urbain de porcs.

Dans un contexte africain où les liens entre la ville et l'agriculture sont en permanence reconstruits et renégociés, l'hypothèse émise ici est que les éleveurs urbains ne sont pas sans moyens. Ils ont des capacités de négociation et d'adaptation et développent des arrangements pour accéder aux ressources essentielles à la conduite de leur activité et assurer les composantes autocentrée et exogène de leur activité. Les agriculteurs doivent «s'arranger » avec le milieu urbain, « innover pour gérer de manière efficace les contraintes urbaines et exploiter aussi efficacement les avantages [...] que procure la ville » (Mougeot, 2000). La notion d'arrangement est employée ici à travers un prisme géographique. Elle est définie comme une négociation entre acteurs, formelle ou informelle, tacite ou explicite, qui vise à régler un problème à dimension spatiale. La dimension spatiale de ce problème tient au fait qu'il s'agit d'une gestion de la distance (accès à une ressource distante, accès à un espace, gestion de la proximité avec le voisinage, gestion de la coprésence d'activités différentes, négociation pour la protection d'un espace, etc.) (Robineau, 2015).

\section{Cas d'étude}

La ville de Bobo-Dioulasso est la deuxième ville du Burkina Faso. Souvent qualifiée de ville agricole, elle abrite un large éventail d'activités agricoles urbaines, des marchés régionaux de produits agricoles et des agro-industries qui fournissent des sous-produits utiles à l'alimentation animale, notamment aux élevages urbains. La proximité des ressources alimentaires (drêche de bière industrielle et traditionnelle, son de maïs, restes de repas) que procure l'environnement urbain a favorisé le développement de l'élevage de porcs en claustration. Après le maraîchage, ce type d'élevage est l'activité agricole urbaine la plus répandue pour générer des revenus. De nombreux éleveurs sont installés soit au sein de l'espace bâti urbain, soit en frange urbaine.

\section{Collecte et analyse des données}

L'étude a été réalisée entre 2010 et 2013 (Robineau, 2013). La collecte de données reposait sur un travail empirique approfondi et s'est faite majoritairement par la conduite d'entretiens compréhensifs et semidirectifs, et par l'étude des réglementations et des plans d'aménagement urbain. Les éleveurs urbains de porcs à petit effectif ont été au centre de l'étude.

Dans un premier temps, une estimation du nombre d'éleveurs de porcs dans la ville et sa frange urbaine a été effectuée. Des entretiens avec des éleveurs ont permis d'identifier les différents profils existants et les localisations des élevages en fonction des contraintes et opportunités socioéconomiques des familles.

Dans un deuxième temps, afin de comprendre la perception de l'élevage de porcs en ville, ainsi que les réglementations et les appuis techniques qui existent, quinze enquêtes ont été réalisées auprès d'élus, d'agents des services techniques municipaux et d'agents d'encadrement agricole. Des discussions informelles avec des citadins de différents quartiers ont permis d'appréhender la vision qu'ont les habitants de la ville de ce type d'élevage.

Enfin, dans un troisième temps, les pratiques des éleveurs ont été analysées en fonction du quartier d'habitat. En effet, selon les caractéristiques sociospatiales des quartiers dans lesquels ils vivent, les arrangements que les éleveurs développent avec d'autres acteurs pour mettre en place leurs pratiques d'élevage diffèrent. Le tissu urbain de Bobo-Dioulasso se différencie en fonction de l'ancienneté et de la « légalité » des quartiers, mais aussi de la mixité sociale de leurs habitants. Quatre grands types de quartiers existent (figure 1) :
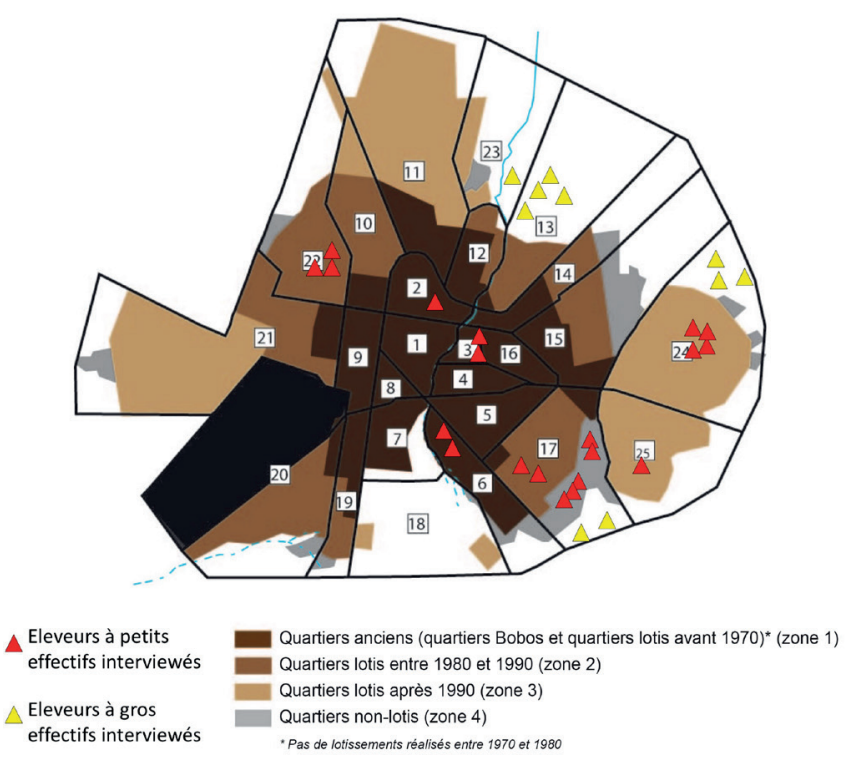

Figure 1 : quatre types de quartiers à Bobo-Dioulasso, Burkina Faso (réalisation : O. Robineau, 2013). 
- les anciens quartiers de Bobo-Dioulasso (zone 1) ont une origine antérieure à 1970. Ils forment des espaces bâtis plutôt denses, localisés dans le centre de la ville, avec des concessions familiales anciennes. Les familles voisines ont soit des liens de parenté, soit elles se connaissent depuis plusieurs générations ;

- les quartiers lotis entre 1980 et 1990 (zone 2) sont des quartiers plus récents, peuplés de familles d'origine différente. On y retrouve des anciens agriculteurs d'ethnie bobo et des anciennes familles des zones d'habitat spontané qui ont été expropriés lors de la création de ces quartiers (appelé processus de lotissement) et qui ont reçu en compensation des parcelles constructibles dans ces mêmes quartiers. Les habitants sont aussi des familles plus aisées qui ont acheté des parcelles d'habitation dans ces quartiers. Les voisins ont des relations assez étroites mais n'ont en général aucun lien de parenté ;

- les quartiers les plus récemment lotis - après 1990 - (zone 3) sont aussi peuplés de familles d'origine différente. Comme dans le cas précédent, on retrouve des Bobo expropriés de leurs champs, des anciens habitants du non loti expropriés lors du lotissement, et des familles plus aisées ayant acheté des parcelles d'habitation dans ces secteurs. Alors que les anciens habitants du non loti se connaissent tous car ils sont d'anciens voisins, les relations de voisinage avec les nouveaux arrivants sont pour l'instant ténues ;

- les quartiers d'habitat spontané, appelés aussi les non lotis (zone 4), regroupent des familles d'origine différente, toutes en situation financière précaire. Certaines familles sont voisines depuis des années et entretiennent des relations étroites. Dans ces zones les lois municipales ne s'appliquent pas, chacun peut donc élever des porcs sans craindre que les plaintes aux services d'hygiènes ne compromettent son activité.

Cinq éleveurs ont été enquêtés dans chaque type de quartiers - soit 20 éleveurs à petit effectif en tout -, ainsi qu'une dizaine d'éleveurs à gros effectif installés en frange urbaine. Dans chaque type de quartier, il s'agissait de partir d'un premier éleveur enquêté et de rencontrer les autres éleveurs auxquels il faisait référence afin de comprendre les réseaux sociotechniques et les arrangements qui existent, les pratiques sur lesquelles ces arrangements portent et la manière dont ils se construisent. Les liens aux voisinages faisaient partie des sujets traités pendant l'enquête.

Les données collectées ont permis de caractériser les arrangements développés pour la conduite des élevages, et de cartographier les réseaux et les lieux des éleveurs de porcs des différents quartiers afin de souligner les conditions de développement et de maintien des élevages urbains de porcs en claustration.

\section{RESULTATS ET DISCUSSION}

\section{Profil des éleveurs de porcs à Bobo-Dioulasso}

En se basant sur les estimations de la Direction provinciale des ressources animales et sur les entretiens effectués dans les différents quartiers (aussi nommés secteurs) de Bobo-Dioulasso, la figure 2 présente l'estimation du nombre d'élevages de porcs par quartier. Il apparaît que des élevages de porcs sont présents dans quasiment tous les quartiers de la ville, sauf dans certains quartiers résidentiels riches (secteurs 5 et 8 ), et dans une moindre mesure dans les quartiers principalement musulmans (secteur 12 par exemple).

A Bobo-Dioulasso, il existe deux grands types d'éleveurs de porcs en claustration. D'une part, des personnes aisées (souvent médecins, fonctionnaires ou commerçants) développent des élevages à gros effectif (au moins cinq truies) pour générer un revenu complémentaire et/ou préparer leur retraite. Ces éleveurs ont eu les moyens d'acheter des terres agricoles en frange urbaine, hors de l'espace bâti, dans des zones favorables à l'élevage, afin de se soustraire aux contraintes de la ville tout en conservant l'avantage de la proximité avec celle-ci.
Leurs élevages sont donc localisés hors de l'espace bâti sur des terres d'environ 0,5 hectare, où se situent à la fois les bâtiments d'élevage et une culture de céréales afin de fournir une partie de l'alimentation des animaux.

D'autre part, la majorité des éleveurs de porcs sont des familles au statut plus précaire, qui développent des élevages à petit effectif (deux truies maximum). Ce sont soit des familles originaires de BoboDioulasso (d'ethnie bobo) n'ayant plus de terres agricoles, soit des migrants de première, seconde ou troisième génération. En l'absence d'opportunités d'emploi en ville, ces familles ont choisi de développer l'élevage de porcs pour générer des revenus. En effet, il s'agit d'un animal prolifique, l'investissement initial est faible, l'élevage peut être conduit sur une surface réduite, et son alimentation repose sur des résidus d'alimentation humaine. Le choix de développer une activité agricole pour générer des revenus s'explique entre autres par le lien encore fort des familles au milieu d'origine rural et au savoir-faire qui en découle, ainsi que par la forte demande en viande porcine à BoboDioulasso qui garantit la vente des animaux. Cet élevage fournit la plus grande part du revenu des familles mais il est souvent combiné à une autre activité (petite activité commerciale, couture, etc.).

Les éleveurs sont installés dans les quartiers lotis (quartiers d'habitat planifié) ou dans les quartiers non lotis (quartiers d'habitat spontané). En effet, leur faible capacité financière ne leur permet pas d'acquérir des terrains en frange urbaine ; ils n'ont donc aucune terre agricole. Ils conduisent leur élevage en claustration dans les cours familiales au sein de la ville. Ainsi, la grande majorité des élevages de porcs qui se situent au sein de l'espace bâti sont des élevages à petit effectif qui comprennent une à deux truies, et parfois un verrat (à ceux-ci s'ajoutent les petits engraissés, à raison de deux mises bas par an par truie, et six à huit petits par mise bas, cela fait une moyenne de 28 porcs engraissés par an). Les box d'élevage sont en briques de terre, étroits, souvent en mauvais état, et ont un toit en tôle ondulée ou en paille pour protéger les animaux du soleil. Bien souvent, depuis la rue, rien ne laisse penser qu'un élevage de porcs se situe à l'intérieur d'une cour. C'est ce deuxième profil d'éleveurs qui est au centre de cet article.

Les éleveurs de porcs sont en majorité des hommes (le chef de famille développe l'élevage) ou des femmes veuves. Les familles des éleveurs sont constituées des parents et des enfants (famille nucléaire).

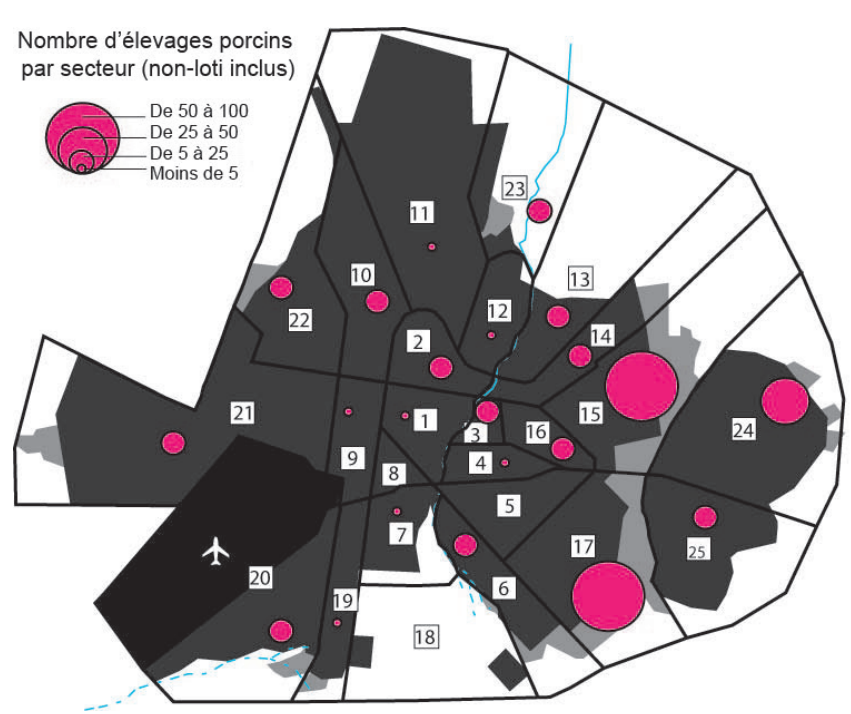

Figure 2 : estimation du nombre d'élevages de porcs par secteur à Bobo-Dioulasso, Burkina Faso, en 2012 (les numéros de secteurs sont encadrés). Source : enquêtes et données de la Direction provinciale des ressources animales (réalisation : O. Robineau, 2012). 


\section{Perception de l'élevage urbain de porcs: vision négative mais tolérance implicite}

Malgré les lois interdisant d'élever en ville, les élus et les agents des services techniques municipaux perçoivent l'élevage urbain - comme bien d'autres activités informelles - comme un des moyens de régler la crise sociale due au manque d'offre d'emploi en ville. Niang (1997) avait mis ceci en évidence à Dakar, dans les années ayant suivi le «Plan d'ajustement structurel » : les activités informelles permettaient d'amortir les conséquences de la crise de l'emploi. En théorie, le service d'hygiène intervient quand des voisins se plaignent de nuisances d'un élevage : en cas de plainte, l'éleveur doit payer une amende et stopper définitivement son activité d'élevage. Mais dans les faits le service d'hygiène intervient plutôt sous forme de négociation, en fonction de la nature de la plainte, afin de permettre à l'éleveur de continuer son activité tout en diminuant les nuisances (sonores et/ou olfactives) pour éviter de nouvelles plaintes.

La tolérance des élus et agents des services techniques municipaux envers les éleveurs de porcs se retrouve aussi chez les citadins. L'élevage de porcs peut déranger, par son odeur et son bruit, mais le voisinage importuné est aussi solidaire de l'éleveur qui a besoin de cette activité pour vivre. Si un voisin se plaint au service d'hygiène, il demande souvent l'anonymat car causer du tort à son voisin est quelque chose de mal perçu dans le pays où « le voisin est le premier parent» (adage burkinabé). Les plaintes aux services d'hygiène ne sont donc pas la norme et sont finalement peu nombreuses, si l'on considère le nombre d'élevages qui existent en ville : « on peut faire un mois sans plainte » (commun. pers., responsable du service d'hygiène). Il est important de souligner qu'un voisin musulman n'est pas forcément plus hostile à l'élevage de porcs qu'un voisin non musulman.

Un encadrement technique existe pour l'élevage de porcs, à travers la Maison des éleveurs de porcs (MEP) qui existe depuis 2001. La MEP fut créée à l'initiative d'éleveurs soutenus par un projet de développement agricole centré sur l'élevage à cycle court. Elle permet notamment aux éleveurs d'accéder à des marchés permettant d'obtenir un prix de vente plus élevé qu'habituellement (avec des clients de Ouagadougou par exemple), et a aussi contribué à introduire de nouvelles races de porcs, telle que la Large White, une race sélectionnée en Europe. Pour pouvoir adhérer à la MEP il faut accepter de faire de l'élevage en claustration, payer une cotisation de 5000 FCFA par an, et les porcs vendus par le biais de la MEP doivent dépasser 80 kilogrammes. Mais les éleveurs de porcs urbains à petit effectif n'ont pas les moyens de payer l'adhésion ni de vendre des porcs de 80 kilogrammes. Souvent, les animaux sont vendus plus tôt, quand la famille a besoin d'argent. Les éleveurs de porcs à petit effectif ne bénéficient donc pas directement de l'appui de la MEP qui a clairement une politique de soutien aux éleveurs (qu'elle qualifie de « professionnels ») à plus gros effectif.

\section{Elever des porcs en ville : gérer la mobilité réduite et la proximité avec le voisinage}

\section{Pratiques d'élevage contraintes par une faible mobilité spatiale}

Les pratiques d'élevage urbain de porcs à petit effectif reposent sur une logique de mobilité spatiale réduite. Circuler se fait au minimum, à l'image du porc qui est un " piètre marcheur », qui « ne s'épanouit que dans un milieu lui offrant suffisamment de nourritures riches en protéines dans un rayon restreint. Il ne se déplace généralement pas » (Faye et Porphyre, 2011). Dans cette logique, être éleveur porcin urbain repose sur des interactions au sein d'un quartier, voire du voisinage immédiat. Le lieu d'habitat de l'éleveur et son lieu de travail coïncident, et l'accès aux ressources alimentaires pour les animaux se fait dans des lieux proches de la concession familiale.
La mise en place des pratiques d'élevage - de la reproduction à la vente - est soumise à un ensemble de facteurs internes et externes (accès aux aliments, gestion des liens de voisinage) que les éleveurs doivent maîtriser. Il est question ici de la gestion de la reproduction, de l'accès aux aliments et de l'accès à l'information technique. Ces pratiques et leurs contraintes de mise en œuvre sont présentées ci-dessous.

Les éleveurs doivent trouver deux fois par an des mâles reproducteurs. Ils évitent la consanguinité en changeant régulièrement de verrat et doivent gérer le calendrier de reproduction pour échelonner les périodes de mise bas et éviter d'avoir un sureffectif (qui poserait à la fois des problèmes de place dans les box et de disponibilité des ressources alimentaires). Si les éleveurs n'ont pas de verrat et doivent en emprunter un, cela doit se faire à une distance relativement réduite. En effet, les porcs sont peu mobiles et ne peuvent pas marcher de longues distances sous la chaleur, et si le transport nécessite une charrette, alors c'est le coût du transport qui augmente avec la distance.

L'alimentation des porcs est quant à elle une préoccupation quotidienne des éleveurs. Katongole et al. (2013) ont mis en évidence les difficultés qu'éprouvent les éleveurs urbains à trouver les aliments en quantité suffisante pour nourrir leurs animaux. Les deux principaux aliments entrant dans l'alimentation des porcs sont la drêche de dolo (bière traditionnelle) - base de l'alimentation qui fournit l'apport protéique -, et le son de maiis - qui fournit l'apport énergétique. Normalement cette ration doit s'accompagner de minéraux et de sels de cuisine que les éleveurs de porcs à petit effectif n'ont pas souvent les moyens financiers d'acquérir. Les quantités données de manière journalière varient selon la trésorerie de l'éleveur. Un seau de 10 litres de mélange (drêche + son) peut être distribué par jour à chaque truie et ses petits, mais ceci peut baisser jusqu'à un demi-seau si l'éleveur n'a pas les moyens de payer la ration journalière. Si l'éleveur n'a pas de charrette, il doit la transporter à pied ou en vélo. Dans ce cas, seules de petites quantités peuvent être transportées par trajet, et ceci doit se faire quotidiennement, car cet aliment frais et humide ne se conserve pas ; d'où l'intérêt d'avoir un lieu d'approvisionnement à proximité de l'élevage. Dans un contexte où le nombre d'animaux en ville ne cesse d'augmenter, que ce soit des petits, des gros ruminants ou des monogastriques, la demande pour la drêche et le son s'accroît sans cesse, faisant augmenter les prix et la compétition pour l'achat de ces produits.

Aussi, les éleveurs ont besoin d'actualiser leur savoir technique pour améliorer leurs pratiques d'élevage. N'ayant pas les moyens d'adhérer à la MEP, ils trouvent via leur réseau personnel des voies d'accès à l'information technique.

Enfin, un autre aspect sensible de l'élevage de porcs est la gestion des effluents. En effet, les déjections porcines sont nauséabondes et sont la cause de nuisances olfactives importantes. A Bobo-Dioulasso, il n'existe pas de système de gestion des effluents par la ville et chaque éleveur doit donc trouver le moyen de se débarrasser au plus vite de ceux-ci. L'activité maraîchère urbaine étant très développée à BoboDioulasso (plus de 150 hectares de zones maraîchères dans la ville et sa très proche périphérie), la demande en déjections animales est forte : les éleveurs sont très sollicités pour l'achat des déjections porcines. Il existe donc des arrangements entre éleveurs et maraîchers pour la vente de celles-ci. Ces pratiques ne sont pas détaillées dans cet article car elles sont décrites en détail dans celui de Robineau (2015).

\section{Pratiques et lieux d'élevage : importance de la confiance et de la proximité}

Quel que soit le type de quartier dans lequel ils se situent, les éleveurs de porcs à petit effectif ont besoin d'être en interaction forte avec d'autres éleveurs pour conduire leur activité. Ces interactions sont à l'origine du développement d'arrangements qui leur permettent 
d'avoir accès à des ressources externes nécessaires à la conduite de leur élevage. La compréhension des arrangements développés par les éleveurs de porcs des différents quartiers permet d'identifier des invariants dans les conditions d'émergence de ces arrangements. Le cas d'éleveurs de vieux quartiers (zone 1) est d'abord présenté afin d'illustrer comment ceux-ci gèrent la conduite de leur élevage et comment les arrangements qu'ils développent y participent. Ce premier cas est ensuite comparé avec les stratégies et les arrangements d'éleveurs présents dans les autres types de quartiers. Le cas des éleveurs du non loti (zone 4) n'est pas développé spécifiquement ici afin d'éviter les redondances, car les arrangements et leurs conditions de développement sont liés à ceux observés dans les quartiers les plus récemment lotis (zone 3).

- Elever des porcs dans les vieux quartiers :

la proximité des liens forts

Les éleveurs présents dans les vieux quartiers de la ville (zone 1) sont issus de familles résidant depuis des générations dans ces mêmes quartiers. Le plus souvent, environ quatre à cinq éleveurs à petit effectif résident à proximité les uns des autres, à moins de deux rues d'écart (figure 3) : l'un d'entre eux a souvent servi d'exemple aux autres, et ils ont des élevages similaires en termes de taille (une à deux truies et parfois un verrat) et de conduite technique. Pour la conduite quotidienne de leurs élevages, ces éleveurs entretiennent des liens étroits entre eux, à la fois pour les conseils, l'entraide, le soutien moral et la reproduction des animaux. En effet, entretenir un verrat est coûteux, et avoir un seul verrat n'est pas suffisant pour gérer seul la reproduction de ses bêtes sans avoir de problème de consanguinité (des petits sont gardés pour le renouvellement des truies). Quand c'est nécessaire, un éleveur emprunte ainsi un verrat à un éleveur de son entourage. Prêter ou échanger des verrats implique d'avoir confiance en l'autre éleveur car cela engendre un risque à la fois de transmission de maladies, mais aussi que l'éleveur emprunteur ne nourrisse pas bien le verrat pendant la période où il l'accueille dans sa porcherie. Quand les éleveurs se connaissent bien, l'emprunt de verrat est gratuit ; celui qui emprunte a à sa charge l'alimentation du verrat pendant la période d'emprunt, qui peut aller jusqu'à une semaine. Parfois, un éleveur peut accepter de prêter à un éleveur qu'il ne connaît pas, sous réserve qu'il soit l'ami d'un des éleveurs duquel il est proche : dans ce cas l'emprunt peut être payant.

Ces éleveurs essaient d'entretenir des liens avec des éleveurs à plus gros effectif localisés en frange urbaine (figure 3), plus avancés

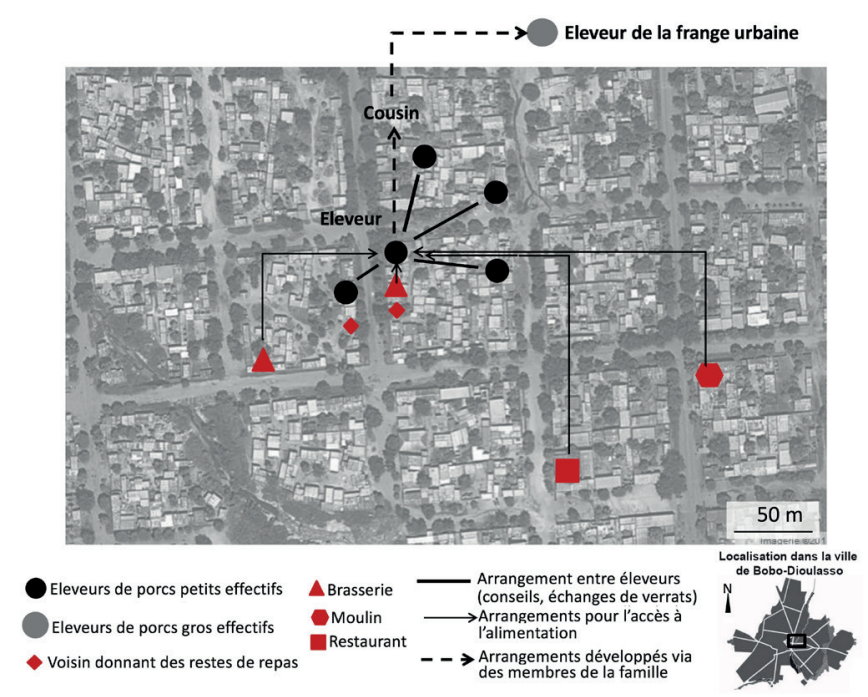

Figure 3 : exemple d'un éleveur de porc d'un vieux quartier de Bobo-Dioulasso, Burkina Faso. Le double ancrage des arrangements, la famille et le voisinage (réalisation: O. Robineau, 2017). techniquement, afin de bénéficier de leurs conseils et éventuellement de leurs verrats de races améliorées. Bien souvent un éleveur du quartier connaît personnellement un éleveur de porcs à gros effectif membre de la MEP car ils ont été voisins. Il est toutefois très rare qu'un éleveur de porcs à gros effectif prête un verrat à un éleveur à petit effectif en raison des pratiques sanitaires plus aléatoires chez les petits éleveurs (la grippe porcine a augmenté la méfiance des éleveurs) et des problèmes de trésorerie auxquels ils font face; les éleveurs à gros effectif refusent de prêter leur verrat pour éviter les risques de maladies et de malnutrition. Cependant, la diffusion de races améliorées indique que quelques prêts entre ces deux types d'éleveurs ont eu lieu. En effet, ce que les éleveurs à petit effectif appellent de «bons verrats » sont des verrats de race améliorée ; les élevages à petit effectif comptent couramment des porcs métis créole x Large White.

Les éleveurs achètent la drêche de dolo dans les brasseries traditionnelles du quartier. Ils la complètent avec du son de maïs, et régulièrement avec des restes de restaurants et des déchets alimentaires des voisins. Les éleveurs collectent ces résidus alimentaires en laissant des seaux dans les restaurants ; les voisins quant à eux apportent spontanément leurs restes alimentaires aux éleveurs. L'accès à quasiment l'ensemble des aliments se fait grâce à des contacts établis via les amis ou la famille. L'acquisition de drêche fait l'objet d'arrangements plus ou moins formalisés sous forme «d'abonnements »- sorte de contrat oral - avec les « dolotières » (fabricantes de dolo). L'éleveur reçoit chaque jour un peu de drêche en fonction de ce qu'il a pu négocier avec la dolotière. Eleveurs et dolotières s'accordent sur les modalités de paiement : l'éleveur paye soit au jour le jour, soit à la fin du mois. Cette deuxième modalité implique d'avoir un niveau de confiance réciproque élevé car le décompte du nombre de seaux fournis durant le mois peut faire l'objet de litiges. Dans certains cas, les éleveurs ne sont pas abonnés, mais les relations très anciennes avec une dolotière font qu'elle lui réserve la drêche chaque jour, tout comme dans le cas d'un abonnement.

Ces relations à trois niveaux (avec les éleveurs voisins, avec les fournisseurs d'intrants / revendeurs et avec les éleveurs techniquement plus avancés) se retrouvent chez l'ensemble des éleveurs de porcs urbains à petit effectif rencontrés, quel que soit le type de quartier. Ce qui diffère, c'est l'ancrage spatial et la construction des liens.

- Etre éleveur dans un quartier loti après 1980 :

le continuum social du quartier d'origine

Que ce soit dans les quartiers lotis entre 1980 et 1990 (zone 2) ou dans les quartiers lotis après 1990 (zone 3), les éleveurs restent très attachés à leur quartier d'origine. Dans ces quartiers plus récents, les éleveurs de porcs ont deux origines différentes. Soit il s'agit d'anciens habitants bobo des vieux quartiers centraux, qui ont perdu leurs champs lors des lotissements ; une parcelle constructible leur a été attribuée en compensation et ils ont décidé d'aller vivre dans ce nouveau quartier en raison du manque de place dans la cour familiale d'origine (Robineau et Dugué [2017] expliquent notamment les dynamiques foncières et les mécanismes d'attribution des parcelles). Soit il s'agit de familles installées dans le quartier depuis que celui-ci a été loti ; elles habitaient dans une zone non lotie et une parcelle leur a été attribuée lors de l'opération de lotissement. Le nombre d'élevages de porcs est moins élevé dans ces quartiers que dans les vieux quartiers majoritairement catholiques de la ville mais les éleveurs qui se côtoient sont géographiquement proches, seules deux ou trois rues les séparent.

Les éleveurs originaires des vieux quartiers et installés dans les quartiers lotis après 1980 gardent des liens forts avec leur quartier d'origine, dans lequel vit toujours leur famille élargie. Ils côtoient à la fois un ou deux éleveurs résidant dans leur nouveau quartier (mais originaire du même quartier qu'eux), et deux ou trois éleveurs localisés dans leur quartier d'origine, comme l'illustre l'exemple des éleveurs E. et F. (figure 4). Les liens entre éleveurs sont donc à la fois ancrés dans le temps et liés à un quartier d'origine commun. Comme dans 
le cas précédent, l'un d'entre eux connaît un éleveur de la MEP avec qui il est en contact régulièrement quand il a besoin d'une information technique que ses voisins éleveurs ne peuvent pas lui procurer. Aussi, comme pour les éleveurs des vieux quartiers, l'accès à l'alimentation pour les porcs se gère dans un périmètre proche. Ces éleveurs ont pu s'abonner auprès de dolotières pour obtenir de la drêche de dolo suite à une fréquentation régulière des brasseries traditionnelles, qui a permis d'établir une relation de confiance avec les dolotières. Contrairement aux vieux quartiers, ce ne sont pas les liens familiaux qui ont facilité la création de ces « contrats » pour l'achat des aliments, mais une fréquentation répétée du lieu.

Les relations des éleveurs originaires du non loti (zone 4) et qui résident et élèvent aujourd'hui des porcs dans les quartiers lotis après 1990 (zone 3) sont similaires à celles mentionnées ci-dessus. La principale différence tient aux origines des liens entretenus entre éleveurs. Le non loti tient le rôle du quartier d'origine, qui n'existe plus physiquement et qui a été remplacé par un quartier loti, mais dont les relations sociales perdurent au-delà de la restructuration spatiale du quartier.

Le cas d'A.,éleveur dans le secteur 24, permet d'illustrer ceci (figure 5). Lors de la destruction de la zone non lotie où vivait A., il a été attributaire d'une parcelle au sein du nouveau quartier bâti sur l'ancien non loti, et a ainsi pu conserver son ancienne maison : il y vit toujours avec sa femme et possède une porcherie de trois box juste devant celle-ci. A. a commencé l'élevage de porcs alors que ce quartier était encore non loti. Lui et trois de ses voisins ont suivi l'exemple de T., un voisin qui élevait des porcs depuis quelques années et possédait déjà plus de cinq truies. C'est à lui qu'A. a acheté sa première truie. Lors du lotissement, la plupart ont continué d'élever des porcs dans leur cour familiale. Malgré la restructuration du quartier, ils sont restés voisins (figure 5). Seul T., qui disposait de revenus plus importants, a décidé d'acheter une parcelle en frange urbaine pour y déménager et installer son élevage qui compte aujourd'hui 10 truies. Même s'ils ne sont plus voisins, il est resté le contact technique d'A. et de ses voisins éleveurs, car il a l'expérience et il est adhérent à la MEP. A. achète la drêche de dolo chez une dolotière qui brassait de la bière dans ce même lieu حิ avant le lotissement; elle met de côté quotidiennement ce dont il a besoin. A. garde aujourd'hui des contacts rapprochés avec les éleveurs qu'il connaît depuis l'avant-lotissement et ceux localisés à moins de deux ou trois rues de chez lui. Au-delà, il ne connaît pas directement les éleveurs s'il n'a pas été leur voisin dans le passé. L'échange de verrats se fait principalement avec les voisins qu'il connaît depuis l'avantlotissement. T. ne prête qu'exceptionnellement des verrats pour éviter les maladies et les problèmes potentiels d'alimentation.

\section{Maintenir son élevage en ville : quand le voisinage est la clé}

Elever des porcs en ville n'implique pas seulement la conduite technique de l'activité elle-même. Cela implique aussi de la conduire dans des conditions sociospatiales spécifiques, plus ou moins favorables à la présence d'élevage. Les différentes caractéristiques des quartiers décrites plus haut interrogent la manière dont les éleveurs parviennent à s'insérer dans les quartiers, et la manière dont les relations de voisinage avec les résidents non éleveurs peuvent être une condition du maintien ou non de l'activité d'élevage à court, moyen et long terme.

Dans les vieux quartiers de Bobo-Dioulasso (zone 1), le voisinage est un espace de sécurité. Les familles se connaissent depuis plusieurs générations. Ceci induit une tolérance et une solidarité des uns envers et les autres, qui est ressentie positivement par les éleveurs. Les voisins des éleveurs leur donnent de la nourriture (restes de repas), les encouragent, et les éleveurs tuent régulièrement un porc lors des fêtes. Dans les sociétés africaines, donner, recevoir et redistribuer est une obligation sociale (Mahieu, 1991), et un refus de donner pourrait engendrer une rupture dans les relations familiales et de voisinage. De plus, les habitants du quartier savent que l'activité d'élevage permet de faire vivre leurs voisins ; ce système de don contre don fait partie des liens qui
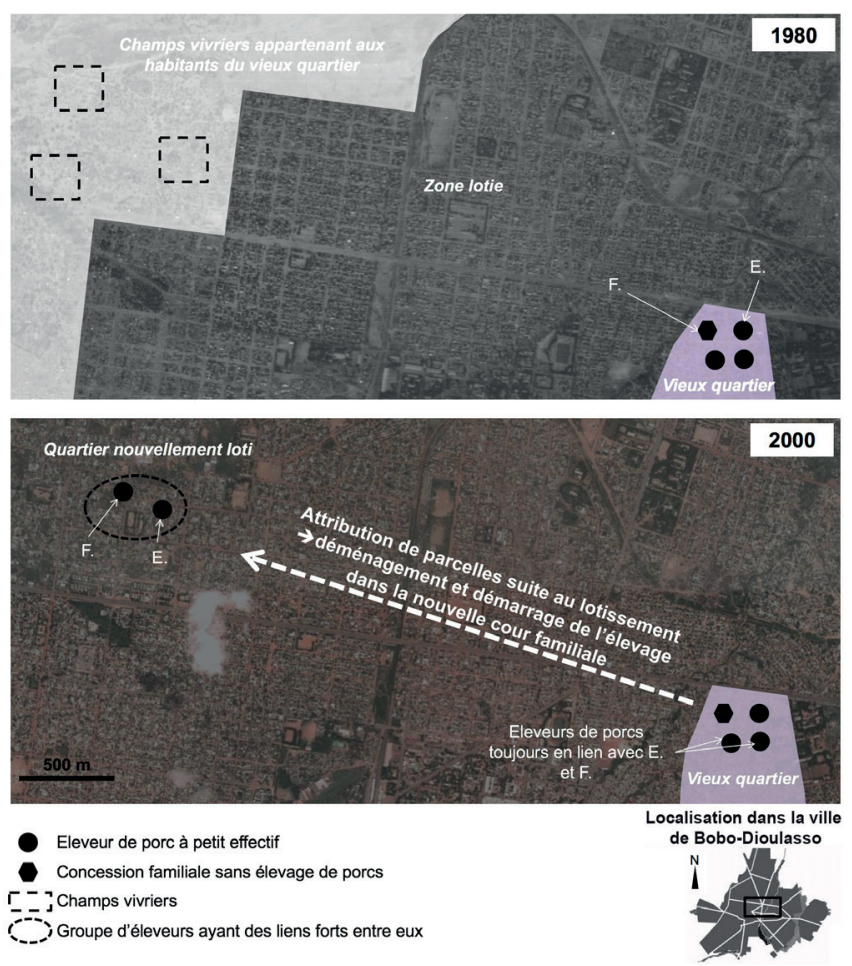

Figure 4 : changement du lieu d'élevage et continuité des arrangements entre éleveurs de porcs à Bobo-Dioulasso, Burkina Faso. Exemple des éleveurs E. et F. (réalisation : O. Robineau, 2017).
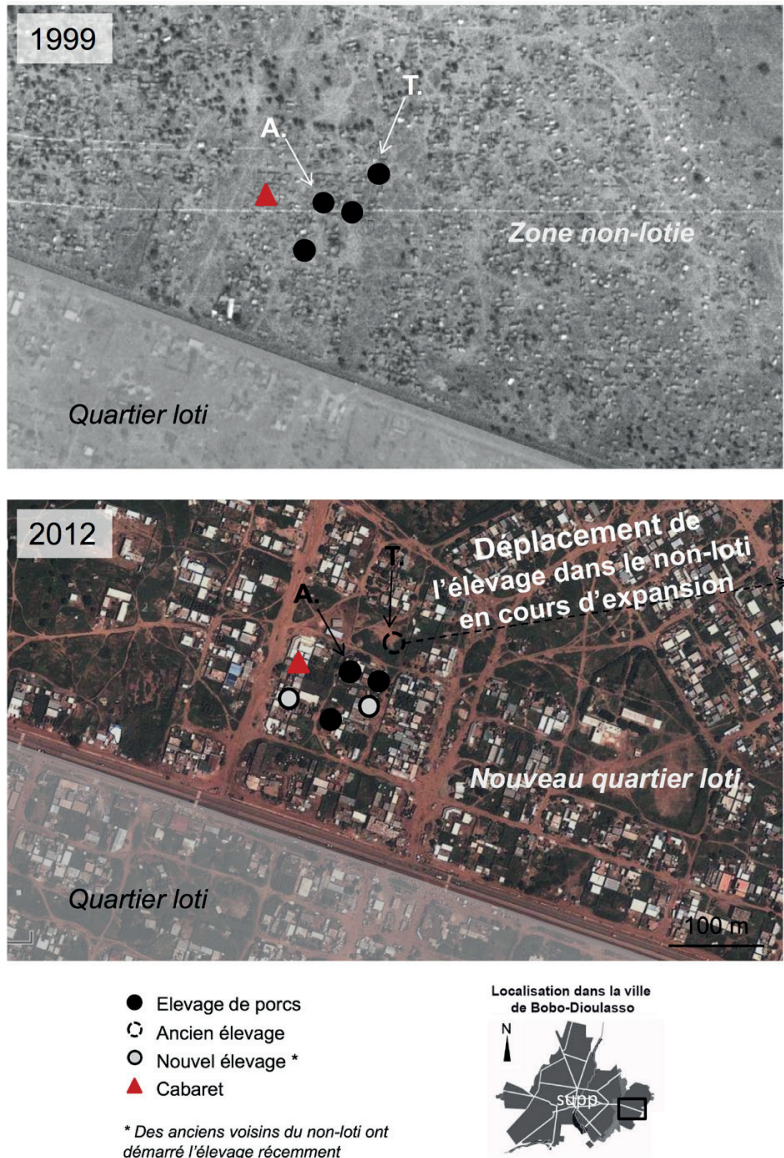

Figure 5 : constance et changements dans le voisinage de l'éleveur A. lors du lotissement du quartier à l'est de Bobo-Dioulasso, Burkina Faso (réalisation : O. Robineau, 2017). 
renforcent l'activité d'élevage. Le quartier est l'espace des liens sociaux forts, familiaux et amicaux, qui procurent une certaine sécurité aux éleveurs, notamment pour l'accès à des ressources alimentaires ou reproductives dans ces quartiers densément peuplés. Cette forte intégration sociale de l'éleveur dans son voisinage se retrouve chez l'ensemble des éleveurs rencontrés dans les vieux quartiers de Bobo-Dioulasso. Par ailleurs, les éleveurs ajustent leurs pratiques en fonction des contraintes de voisinage. Il s'agit d'arrangements tacites pour limiter les nuisances, comme le stockage des déjections dans un trou puis leur vente rapide à un maraîcher urbain, le nettoyage régulier de la porcherie pour éviter les odeurs, ou la construction d'un mur de séparation un peu plus haut entre les cours pour diminuer les nuisances sonores et olfactives.

La principale différence entre les quartiers lotis entre 1980 et 1990 (zone 2), et les quartiers lotis après 2000 (zone 3) tient aux relations avec les voisins non éleveurs. L'intensité des liens qui lient les éleveurs de porcs à leur voisinage est plus faible que celle des vieux quartiers de la ville et la bienveillance de la part du voisinage est limitée. Dans les quartiers lotis entre 1980 et 1990 (zone 2), les relations entre voisins datent de plusieurs décennies : ceci engendre une relation de confiance et d'entente dans le voisinage, favorisant une tolérance à l'égard des éleveurs comme dans les vieux quartiers de la ville. Même si certains voisins se connaissent depuis longtemps, les éleveurs sont prudents avec les nouveaux arrivants car ils sont conscients des risques qu'ils encourent si un voisin se plaint aux services d'hygiène. Ils développent donc des pratiques pour limiter les nuisances sonores et olfactives, avec parfois une diminution de l'effectif d'élevage si le risque de nuisances est trop grand.

Les relations de voisinage dans les quartiers lotis après 2000 (zone 3) sont plus ténues car les voisins se connaissent encore peu. L'origine de la population est très diverse et certains habitants, installés en ville depuis plusieurs générations, ont des activités très éloignées de l'agriculture, ne maintiennent plus de liens avec le village originel et le secteur rural, et peinent parfois à accepter la présence d'élevage de porcs dans la cour voisine. Quand les relations de voisinage ne se passent pas bien, cela peut aller jusqu'à la plainte aux services d'hygiène et à l'interdiction d'élever dans la cour. Il existe des cas où des éleveurs ont été obligés d'arrêter définitivement leur activité dans ces quartiers suite à la plainte de voisins car aucun compromis n'a été possible dans le cadre d'une négociation faisant intervenir l'éleveur, son voisin, et les services d'hygiène. Ceci est plus fréquent dans le cas d'éleveurs isolés (à plus de trois rues d'autres éleveurs) et entourés de villas récentes et modernes. Ainsi, même si l'éleveur maintient des relations sociales fortes avec des éleveurs et des dolotières qui lui permettent de conduire son élevage, son activité peut s'arrêter du jour au lendemain à cause d'une plainte d'un voisin.

Enfin, élever dans les zones d'habitat spontané (zone 4) ne dérange pas moins les voisins que dans les quartiers lotis. Néanmoins, dans ces zones il n'existe pas de risque d'intervention du service d'hygiène puisqu'il s'agit d'un espace hors du cadre réglementaire urbain. Ce n'est qu'en cas de lotissement de la zone que le maintien de l'élevage sera remis en question. Cependant, même si les éleveurs ne craignent pas les plaintes, ils s'attachent à conserver de bonnes relations de voisinage. Celles-ci passent, tout comme dans les quartiers lotis, par des pratiques de gestion de l'élevage et des déchets qui visent à limiter au maximum les nuisances.

\section{ARRANGEMENTS POUR LA CONDUITE DE L'ELEVAGE EN VILLE}

Leétude des différentes situations d'éleveurs montre que les arrangements qui existent sont de nature différente (accord explicite entre amis, arrangements implicites, etc.), concernent des objets divers (échange d'information, échange de mâles reproducteurs, accès à l'alimentation, tolérance, etc.), se font dans des quartiers différents et ont des modalités qui varient de manière subtile (abonnement ou pas au cabaret selon le niveau de confiance, échange gratuit de mâles reproducteurs selon les liens d'amitié et la confiance, etc.). C'est bien à l'échelle de l'individu que ces arrangements sont identifiables et que leurs caractéristiques et leur nuance sont perceptibles. Les résultats soulignent ainsi que les mêmes formes d'arrangements se retrouvent dans les différents types de quartiers et sont de trois niveaux :

- les arrangements liés à la conduite quotidienne de l'élevage sont ancrés dans un voisinage géographiquement et socialement proche, facilement accessible. D'une part, les arrangements entre éleveurs à petit effectif d'un même voisinage permettent d'échanger des mâles reproducteurs, des conseils, et de se soutenir moralement dans l'activité. Ces arrangements sont basés sur un niveau de confiance élevé qui s'est construit dans le temps. D'autre part, les arrangements entre les éleveurs et les fournisseurs d'intrants (ici aliments du bétail) font aussi partie des interactions quotidiennes. Les arrangements avec les dolotières, au cœur de l'accès à l'alimentation, se font par la construction d'une relation de confiance acquise, facilitée par une proximité géographique ;

- les arrangements avec les éleveurs de porcs à gros effectif sont géographiquement et socialement plus lointains, moins fréquents et les liens sont plus ténus. Ils permettent d'avoir accès à d'autres types de ressources ; il s'agit d'informations techniques plus poussées, acquises auprès d'éleveurs faisant partie de cercles professionnels auxquels les éleveurs de porcs à petit effectif n'ont pas accès directement. Les éleveurs de porcs à gros effectif sont ainsi des relais de diffusion d'informations techniques et parfois de nouvelles races. Là aussi, ces relations sont ancrées dans le temps et c'est une relation de voisinage ou une mise en contact via un ami ou de la famille qui permet d'être en lien avec ces éleveurs ; il existe ainsi une certaine solidarité qui est plutôt due à des liens personnels qu'à une solidarité professionnelle ;

- enfin, les arrangements avec le voisinage et les autorités relèvent du maintien même de l'élevage en ville. Il peut s'agir d'arrangement plus tacite, comme la gestion de bonnes relations de voisinage. Ces arrangements peuvent aussi être plus explicites et faire intervenir des acteurs publics. Dans ce cas, il s'agit alors de s'arranger pour contourner la réglementation en place afin de permettre aux élevages de perdurer au sein des quartiers habités.

La pratique de l'élevage de porcs urbains repose sur la combinaison de « savoir faire » (connaissances techniques), de «pouvoir faire » (contraintes internes et externes) mais aussi de la composante « avec qui le faire » (relations sociales). Dans un espace urbain a priori défavorable à l'élevage de porcs et pour des éleveurs disposant de moyens financiers réduits l'aspect « avec qui le faire » est central pour la conduite d'un élevage hors-sol qui dépend de ressources externes pour se pérenniser. Ceci permet d'assurer une durabilité à la fois autocentrée (pour la conduite technique de l'élevage) et une durabilité exogène (pour assurer son maintien au-delà des aspects techniques) (figure 6). Les éleveurs de porcs à petit effectif sont au cœur d'un ensemble d'interactions sociospatiales multiformes. Ces interactions, qui prennent la forme d'arrangements entre acteurs, sont principalement développées au sein d'un espace géographique réduit, le voisinage. Au-delà de cette échelle locale, les relations que les éleveurs entretiennent sont principalement liées à des réseaux au sein d'un quartier d'origine et à des réseaux techniques plus lointains (avec les éleveurs de porcs à gros effectif). Ces réseaux de relations fonctionnent en « groupes informels d'éleveurs » liés par une relation de confiance ancrée dans le temps et dans un espace local. Ceci corrobore les résultats de travaux sur l'économie de la confiance (Tazdaït, 2008 ; Laurent, 2012), notamment les travaux sur le fonctionnement de filières animales en Afrique de l'Ouest (Dièye et al., 2008; Tiotsop et al., 2014), qui soulignent l'importance de la confiance acquise dans 


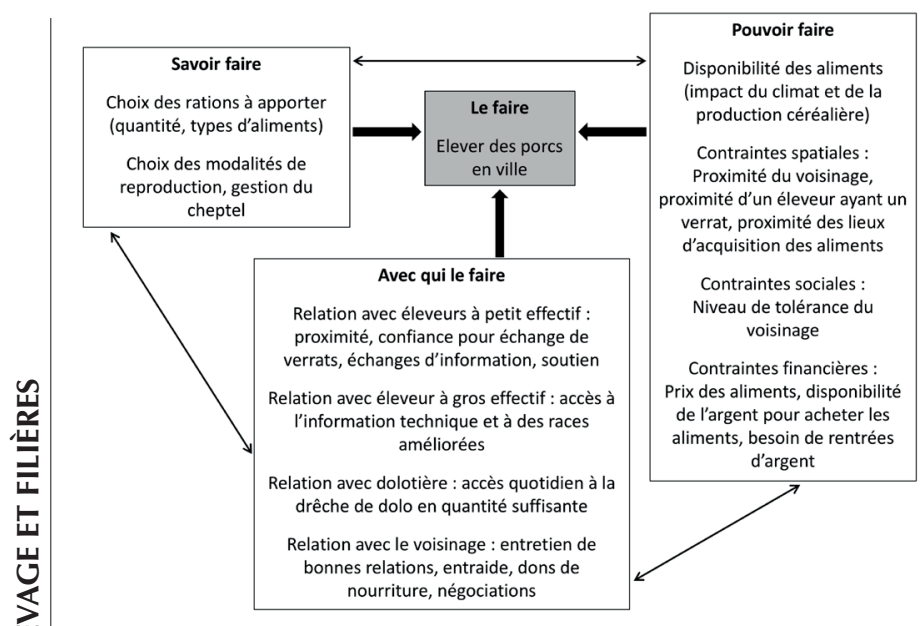

Figure 6 : élever des porcs à Bobo-Dioulasso, Burkina Faso ; "le faire ", combinaison de "savoir faire ", de "pouvoir faire " et $d^{\prime}$ " avec qui le faire " (conception et réalisation : O. Robineau, 2017).

les transactions. L'identification de l'existence de groupes informels à Bobo-Dioulasso souligne que l'élevage urbain de porcs ne peut pas se conduire de manière isolée et qu'une proximité spatiale et sociale entre éleveurs est nécessaire pour assurer la pérennité de ces élevages.

Les résultats amènent à discuter la façon dont l'élevage de porcs est a priori interdit en ville (du point de vue des règlements) mais toléré, et d'une certaine façon rendu invisible. Sa mise en invisibilité participe à son maintien ; en d'autres termes, il s'agit de sa durabilité exogène. Par des arrangements informels, un ensemble d'acteurs participe à cette mise en invisibilité à la fois spatiale (porcs élevés en claustration dans les cours, cachés de la rue, ou installés dans le non loti « illégal »), politique (contournement des lois d'interdiction d'élever en ville, tolérance des élus et des agents municipaux vis-à-vis de cette activité) et sociale (solidarité du voisinage, pratiques de l'éleveur pour limiter les nuisances). La tolérance non affichée est au cœur des négociations qui peuvent exister.

Un dernier élément de discussion porte sur le poids d'acteurs individuels dans le maintien de cette forme d'agriculture urbaine. Cette recherche a permis de mettre en évidence que ce ne sont pas tant les instruments politiques qui contraignent l'agriculture urbaine (par exemple l'arrêté interdisant l'élevage de porcs), mais la manière dont ils sont interprétés, négociés ou contournés par les acteurs institutionnels et/ou par les agriculteurs. On rejoint ici ce que Nuttal et Mbembe (2008) décrivent comme des entremêlements du formel et de l'informel, caractéristiques des villes africaines. Les sensibilités personnelles de certains acteurs institutionnels sont centrales dans ce processus : l'exemple du chef du Service d'hygiène qui négocie la présence de l'élevage en ville est révélateur de ce phénomène. En effet, le maintien des élevages de porcs en ville pose question vis-àvis des règles d'hygiène et de la réglementation existante : sans cette tolérance du service d'hygiène, qui en quelque sorte protège l'élevage de porcs, y-aurait-il autant d'éleveurs de porcs urbains ?

\section{- CONCLUSION}

Alors que l'urbanisation questionne la pérennité de l'agriculture urbaine (La Rosa et al., 2014), celle-ci est de plus en plus acceptée en raison du rôle positif qu'elle joue dans l'approvisionnement alimentaire des villes, la réduction de la pauvreté ou l'intégration sociale de ménages pauvres (Zasada, 2011). La place de l'élevage urbain reste cependant soumise à de nombreuses interrogations sur ses risques sanitaires, et ce type d'activité agricole est beaucoup moins documenté que le maraîchage. Dans ce contexte, l'élevage urbain de porcs fait partie des grands oubliés. Pourtant, il est l'une de ces activités agricoles urbaines qui se développent et font vivre des milliers de familles dans les villes du Sud, malgré les réglementations sanitaires existantes, et les contraintes techniques et sociales liées à la conduite d'un élevage en milieu urbain. Dans un contexte où les données sur l'agriculture urbaine sont encore trop peu nombreuses, il est urgent de dépasser les approches techniques et d'élargir la recherche pour comprendre comment des activités perdurent en ville (Soulard et Aubry, 2011 ; Robineau et al., 2014 ; Robineau et Soulard, 2017).

Le cas d'une ville d'Afrique de l'Ouest présenté ici a permis de souligner la manière dont les éleveurs urbains de porcs gèrent l'accès à des ressources externes, notamment l'alimentation, les mâles reproducteurs et l'information technique, afin de développer et de maintenir leurs élevages en ville. L'analyse de l'environnement sociospatial dans la conduite de l'activité d'élevage et de la perception de ces élevages par les acteurs publics a mis en évidence l'influence d'aspects exogènes dans le maintien de ces élevages. Les éleveurs doivent à la fois gérer la coprésence entre l'élevage et le voisinage urbain, mais aussi trouver les moyens de relier des acteurs et des activités en s'adaptant aux contraintes d'alimentation (élevage hors-sol pour lequel tous les aliments doivent être achetés) et de mobilité limitée qu'impose ce type particulier d'élevage. Enfin, cet article sur la pratique d'une activité illégale en ville permet de souligner que les leviers de maintien de l'agriculture tiennent en partie à des instruments qui existent mais surtout aux acteurs qui les mobilisent.

\section{REFERENCES}

Amadou H., Dossa L.H., Lompo D.J.P., Abdulkadir A., Schlecht E., 2012. A comparison between urban livestock production strategies in Burkina Faso, Mali and Nigeria in West Africa. Trop. Anim. Health Prod., 44 (7): 16311642, doi: 10.1007/s11250-012-0118-0

Aubry C., Ramamonjisoa J., Dabat M-H., Rakotoarisoa J., Rakotondraibe J., Rabeharisoa L., 2008. L'agriculture à Antananarivo (Madagascar) : une approche interdisciplinaire. Nat. Sci. Soc., 16 (1) : 2335

Boussini H., Traore A., Tamboura H.H., Bessin R., Boly H., Ouedraogo A., 2012. Prévalence de la tuberculose et de la brucellose dans les élevages bovins laitiers intra-urbains et périurbains de la ville d'Ouagadougou au Burkina Faso. Rev. Sci. Tech. Off. Int. Epizoot., 31 (3) : 943951

Cantor K.M., 2010. Agricultura urbana : elementos valorativos sobre su sostenibilidad. Cuad. Desarro. Rural, 7 (65) : 6187

Chaléard J.L., Dao The Anh, Huamantico A., Koffi-Didia M., Mesclier E., Monin E., Moustier P., Ninot O., 2014. Spécificités de systèmes de production agricole et d'élevage à la périphérie des métropoles du Sud. In : Métropoles au Sud, le défis des périphéries? (dir. Chaléard J.L.). Karthala, Paris, France, 225-241

Dieye P.N., Montaigne E., Duteurtre G., Boutonnet J.-P., 2008. Le rôle des arrangements contractuels dans le développement du système laitier local et des mini-laiteries au Sénégal ». Econ. rurale, 303-305 : 108-122

Faye B., Porphyre V., 2011. Le dromadaire et le cochon : deux visions opposées de l'élevage ? Nat. Sci. Soc., 19 (4) : 365374.

Godard O., Hubert B., 2002. Le développement durable et la recherche scientifique à l'INRA : rapport à madame la Directrice générale de l'INRA. Rapport intermédiaire de mission, 23 déc. 2002. INRA, Paris, France

Gomgnimbou A.P.K., Nacro H.B., Sanon O.H., Kiendrebeogo T., Sedogo M.P., Martinez J., 2014. La gestion des déjections animales dans la zone périurbaine de Bobo-Dioulasso (Burkina Faso) : structure des élevages, perception de leur impact environnemental et sanitaire, perspectives. Cahiers Agric., 23 (6) : 393-402, doi: 10.1684/agr.2014.0724

ILRI, 2012. Livestock in the city: New study of 'farm animals' raised in African cities yields surprising results. ILRI news, www.ilri.org/ilrinews/index.php/ archives/9563 (consulté le 4 mars 2013) 
Katongole C.B., Nambi-Kasozi J., Lumu R., Bareeba F., Presto M., Ivarsson E., Lindberg J.E., 2012. Strategies for coping with feed scarcity among urban and peri-urban livestock farmers in Kampala, Uganda. J. Agric. Rural Develop. Trop. Subtrop., 113 (2): 165174

Kiendrebeogo T., Mopate Logtene C.Y., Konkobo Y.C., Kabore-Zoungrana C.Y., 2012. Approvisionnement en porcs vifs et viande porcine de la ville de Bobo-Dioulasso (Burkina Faso). J. Agric. Environ. Int. Dev., 106 (2) : 105122

La Rosa D., Barbarossa L., Privitera R., Martinico F., 2014. Agriculture and the city: a method for sustainable planning of new forms of agriculture in urban contexts. Land Use Pol., 41: 290-303, doi: 10.1016/j. landusepol.2014.06.014

Laurent E., 2012L. Economie de la confiance. La Découverte, Paris, France, $124 \mathrm{p}$

Mahieu F.-R., 1991. Les fondements de la crise économique en Afrique. L'Harmattan. Paris, France, 202 p.

Mopaté L.Y., Koussou M.O., Kaboré-Zoungrana C.Y., 2006. Dynamique de la production porcine à N'Djaména (Tchad) : évolution des abattages, des poids carcasses durant cinq décennies et prévisions actuelles. Rev. Sci. Tchad, 9 (2) : 60-70

Mougeot L.J.A., 2000. Urban agriculture: definition, presence, potential and risks. In: Growing cities growing food: Urban agriculture on the policy agenda: A reader on urban agriculture. ETC, DES, Feldafing, Germany, 99117

Niang A., 1997. Secteur informel en milieu urbain, un recours à la crise de l'emploi. Ajustement structurel et emploi au Sénégal. Codesria, Dakar, Sénégal

Nuttall S., Mbembe A. (Eds.), 2008. Johannesburg: the elusive metropolis. Duke University Press, Durham, NC, USA, 398 p., doi: 10.1215/9780822381211
Robineau O., 2013. Vivre de l'agriculture dans la ville africaine. Géographie des arrangements entre acteurs à Bobo-Dioulasso, Burkina Faso. Thèse Doct., Université III, Montpellier, France, 352 p.

Robineau O., 2015. Toward a systemic analysis of city-agriculture interactions in West Africa: a geography of arrangements between actors. Land Use Pol., 49: 322-331, doi: 10.1016/j.landusepol.2015.08.025

Robineau O., Dugué P., 2017. A socio-geographical approach to the diversity of urban agriculture in a West African city. Landsc. Urban Plan., 170: 4858, doi: 10.1016/j.landurbplan.2017.09.010

Robineau O., Soulard C.-T., 2017. Comprendre la complexité des liens villeagriculture : intérêt d'une approche par le système agri-urbain. Le cas de Bobo-Dioulasso, Afrique de I'Ouest. Nat. Sci. Soc., 25 (1) : 36-47, doi: $10.1051 / \mathrm{nss} / 2017013$

Robineau O., Tichit J., Maillard T., 2014. S'intégrer pour se pérenniser : pratiques d'agriculteurs urbains dans trois villes du Sud. Espaces Soc., 158 (3) : 83-100, doi: 10.3917/esp.158.0083

Soulard C.-T., Aubry C., 2011. Cultiver les milieux habités : quelle agronomie en zone urbaine? Agron. Environ. Soc., 1 (2) : 89-101

Tazdaït T., 2008. L'analyse économique de la confiance. De Boeck, Bruxelles Belgique, $180 \mathrm{p}$

Tiotsop F., Guillotreau P., Rouchier J., 2014. Le rôle de la confiance dans les relations d'échange : le cas du marché de poisson de Kribi. Rev. Fr. SocioEco., 14 (2) : 189-220, doi: 10.3917/rfse.014.0189

Zasada I., 2011. Multifunctional peri-urban agriculture - A review of societal demands and the provision of goods and services by farming. Land Use Pol., 28 (4): 639-648, doi: 10.1016/j.landusepol.2011.01.008

\section{Summary}

Robineau O. Rearing pigs in a West African city: Arrangements between actors to manage the proximity between the city and livestock farming

Whereas an increasing number of studies are focusing on urban agriculture, urban pig rearing remains largely unknown. Yet it is present in many cities in the South and provides an income for many families. This study aimed to overpass analyses focusing on technical aspects of this type of farming to take a broader interest in the social and spatial framework in which pig farmers breed pigs in the city. It was based on an empirical approach that made it possible to understand the conditions for the development and maintenance of pig breeding in Burkina Faso's second-largest city, Bobo-Dioulasso. The practices of small-scale livestock breeders are discussed, in particular the part played by the arrangements between actors in mobilizing both the technical and social resources needed to conduct and sustain this type of farming. The results highlight that pig farmers have a logic of reduced mobility: the arrangements they develop with other actors are based on the political, spatial and social invisibility of livestock farming, and the links they develop to access external resources are rooted in time and in close proximity. Their social and spatial environment is central for the daily management of livestock farms as well as for their sustainability in the short and medium terms.

Keywords: swine, backyard raising, urban agriculture, agricultural practices, animal feeding, Burkina Faso

\section{Resumen}

Robineau O. Cría de cerdos en una ciudad de África del Oeste: arreglos entre actores para gestionar la proximidad entre las fincas ganaderas y la ciudad

Mientras que un número creciente de estudios se concentra en la agricultura urbana, la cría urbana de cerdos permanece ampliamente desconocida. Sin embargo, está presente en muchas ciudades en el Sur y provee un ingreso para muchas familias. Este estudio tuvo como objetivo ir más allá los análisis centrados en los aspectos técnicos de este tipo de agricultura para dar un mayor interés al marco social y espacial en el que los finqueros crían cerdos en la ciudad. Está basado en un enfoque empírico que hizo posible la comprensión de las condiciones para el desarrollo y el mantenimiento de la cría de cerdos en la segunda ciudad más grande de Burkina Faso, Bobo-Dioulasso. Se discuten las prácticas de los criadores de ganado a pequeña escala, en particular la parte que juegan los arreglos entre actores en cuanto a movilizar tanto los recursos técnicos, como sociales para conducir y mantener este tipo de producción. Los resultados indican que los criadores de cerdos siguen una lógica de movilidad reducida: los arreglos que desarrollan con otros actores se basan en la invisibilidad política, espacial y social de la producción ganadera y los vínculos que desarrollan para acceder a los recursos externos se encuentran enraizados en tiempo y cercanía. El ambiente social y espacial es central para el manejo diario de las fincas ganaderas, así como para su sostenibilidad a corto y mediano plazo.

Palabras clave: cerdo, explotación en pequeña escala, agricultura urbana, prácticas agrícolas, alimentación de los animales, Burkina Faso 
